\title{
Wavelet-Galerkin Quasilinearization Method for Nonlinear Boundary Value Problems
}

\author{
Umer Saeed and Mujeeb ur Rehman \\ School of Natural Sciences, National University of Sciences and Technology, Sector H-12, Islamabad, Pakistan \\ Correspondence should be addressed to Umer Saeed; umer.math@gmail.com
}

Received 25 February 2014; Revised 26 May 2014; Accepted 26 May 2014; Published 16 June 2014

Academic Editor: Ferhan M. Atici

Copyright ( 2014 U. Saeed and M. ur Rehman. This is an open access article distributed under the Creative Commons Attribution License, which permits unrestricted use, distribution, and reproduction in any medium, provided the original work is properly cited.

A numerical method is proposed by wavelet-Galerkin and quasilinearization approach for nonlinear boundary value problems. Quasilinearization technique is applied to linearize the nonlinear differential equation and then wavelet-Galerkin method is implemented to linearized differential equations. In each iteration of quasilinearization technique, solution is updated by waveletGalerkin method. In order to demonstrate the applicability of proposed method, we consider the various nonlinear boundary value problems.

\section{Introduction}

The Galerkin method [1] is a very well-known method for finding the numerical solutions of differential equations. According to wavelet-Galerkin method, connection coefficients are the inner products of Daubechies scaling functions and their derivatives, because we are taking Daubechies scaling functions as a Galerkin basis. The exact and explicit representations of the differential operators in orthonormal bases of compactly supported wavelets are described by Beylkin [2] and he also discussed the sparse representations of shift operators in orthonormal bases of compactly supported wavelets. Latto et al. [3] gave the connection coefficients for zeroth level of resolution; these connection coefficients are essentially based on an unbounded domain. Chen et al. [4] provided the way of calculating the connection coefficients on a bounded interval and these finite integrals play a vital role in the wavelet-Galerkin approximation of differential equations. Restrepo and Leaf [5] reviewed the inner products of Daubechies wavelets and their derivatives and used the connection coefficients for approximation of differential operators.

Amaratunga et al. [6] implemented the wavelet-Galerkin technique for solving the one-dimensional counterpart of Helmholtz's equation. Mishra and Sabina [7] used the wavelet-Galerkin method for solving linear, homogeneous boundary value problems with constant coefficients and compared the obtained solution with the exact solution by using a family of Daubechies wavelets and at different levels of resolution. Daubechies scaling functions as the Galerkin bases were used by Jianhua et al. [8] and the authors implemented the wavelet-Galerkin method for differential equations with a boundary layer. In order to implement the wavelet-Galerkin method, they considered the linear, inhomogeneous second order boundary value problem with constant coefficients. In [9-13] several applications of wavelet-Galerkin method are done and these applications are for linear boundary value problems. Motivated by the work of authors [6-13], we extended the wavelet-Galerkin method for the solution of nonlinear boundary value problems.

The quasilinearization approach was introduced by Kalaba and Bellman $[14,15]$ as a generalization of the NewtonRaphson method [16] to solve individual or systems of nonlinear ordinary and partial differential equations. The quasilinearization approach is suitable for a general nonlinear ordinary or partial differential equations of any order.

Jiwari [19] used a uniform Haar wavelet method with quasilinearization technique for the approximate solution of Burgers' equation and compared the results with the solutions obtained by the other numerical methods and the exact solution. The same approach was used by Kaur et al. [20] for the solutions of nonlinear boundary value 
problems in which they treated the quadratic nonlinearity of unknown function. In [21], we extend the Haar wavelet quasilinearization technique for fractional nonlinear initial and boundary value problems.

In this paper, we consider the compactly supported Daubechies scaling functions [22] as a Galerkin basis and propose a numerical method by combining wavelet-Galerkin method with quasilinearization technique for solving nonlinear boundary value problems. The method deals with not only quadratic nonlinearities but also various other forms of nonlinearities. To the best of our knowledge, the wavelet-Galerkin method, with Daubechies scaling functions as Galerkin basis, has not been implemented for numerical solutions of nonlinear differential equations. Illustrative problems show the advantage of the proposed method.

The paper is arranged as follows: in Section 2, we give a brief review of Daubechies' wavelets, while in Section 3 we describe the two-term connection coefficients. In Section 4 , we give the procedure of implementation of the waveletGalerkin method. In Section 5, the quasilinearization technique for dealing with ordinary differential equations is discussed, while in Section 6 we apply the wavelet-Galerkin method with quasilinearization technique to some nonlinear boundary value problems. Finally, in Section 7 we conclude our work.

\section{Daubechies' Wavelets}

Daubechies [22, 23] constructed a family of compactly supported orthonormal wavelets. A wavelet system consists of a scaling function $\phi(x)$ and a wavelet function $\psi(x)$. There are two important relations in wavelet theory, which we called two-scale relation. Consider

$$
\phi(x)=\sum_{l=0}^{N-1} p_{l} \phi_{l}(2 x)
$$

and the equation

$$
\psi(x)=\sum_{l=2-N}^{1}(-1)^{l} p_{1-l} \phi_{l}(2 x)
$$

where $\phi_{l}(\cdot):=\phi(\cdot-l)$. Relations (1) and (2) are known as refinement relations. The coefficients $p_{l}$ are called the wavelet filter coefficients. Relations (1) and (2) are also called refinement relations and $N$ (an even integer) is the number of wavelet filter coefficients in the refinement relations. The supports of the scaling function $\phi(x)$ and wavelet function $\psi(x)$ are $[0, N-1]$ and $[1-N / 2, N / 2]$, respectively. Daubechies [22] constructed wavelet filter coefficients $p_{l}$ to satisfy the certain conditions. These conditions are linked with certain properties of scaling and wavelet functions [4]. Consider

$$
\begin{gathered}
\sum_{l=0}^{N-1} p_{l}=2 \Longrightarrow \int_{-\infty}^{\infty} \phi(x) d x=1, \\
\sum_{l=0}^{N-1} p_{l} p_{l-2 m}=2 \delta_{0, m} \Longrightarrow \int_{-\infty}^{\infty} \phi(x) \phi(x-m) d x=\delta_{0, m}
\end{gathered}
$$

$$
\sum_{l=2-N}^{1}(-1)^{l} p_{1-l} p_{l-2 m}=0 \Longrightarrow \int_{-\infty}^{\infty} \phi(x) \psi(x-m) d x=0
$$

for any integer $m$,

$$
\begin{array}{r}
\sum_{l=0}^{N-1}(-1)^{l} l^{m} p_{l}=0 \Longrightarrow \int_{-\infty}^{\infty} x^{m} \psi(x) d x=0 \\
m=0,1, \ldots, \frac{N}{2}-1 .
\end{array}
$$

Relation (3) shows that scaling functions have unit area and relations (4) and (5) indicate the orthonormality of $\phi$ and orthogonality of $\phi$ and $\psi$, respectively. Relation (6) shows that $m$ th moment of $\psi$ is zero; that is, it has $m$ vanishing moments, which implies that we can express the elements of the set $1, x, \ldots, x^{N / 2}-1$ as a linear combination of $\phi(x-k)$, integer translate of $\phi(x)$.

Daubechies wavelet has no explicit expressions for the scaling function $\phi(x)$ and the wavelet function $\psi(x)$ at arbitrary $x$.

The simplest Daubechies wavelet [24] is the Haar wavelet, which has explicit expression for calculating the scaling function $\phi(x)$. It is also called D2, which means the Daubechies wavelet having two filter coefficients. It is also called db1, which means the Daubechies wavelet having one vanishing moment. db2 (D4) has four wavelet coefficients, that is, $N=4$, and two vanishing moments. Similarly, DN has $N$ coefficients and $N / 2$-vanishing moments.

\section{Two-Term Connection Coefficients}

In the present work, we are dealing with the second order nonlinear boundary value problems. We will be concerned with the 2-term connection coefficients. Two-term connection coefficients are defined as

$$
\Omega_{k}^{d_{1}, d_{2}}=\int_{-\infty}^{\infty} \phi^{d_{1}}(x) \phi^{d_{2}}(x-k) d x .
$$

Take $d$ times derivative of the Daubechies scaling function (1), by assuming that it is $d$ times differentiable, to obtain

$$
\phi^{d}(x)=2^{d} \sum_{l=0}^{N-1} p_{l} \phi_{l}^{d}(2 x), \quad \text { where } \phi^{d}(x):=\frac{d^{d} \phi}{d x^{d}} .
$$

Use (8) in (7) and changing variables, to obtain [3]

$$
\Omega_{k}^{d_{1}, d_{2}}=2^{d_{1}+d_{2}-1} \sum_{m, l} p_{m} p_{l-2 k+m} \int_{-\infty}^{\infty} \phi^{d_{1}}(x) \phi^{d_{2}}(x-l) d x .
$$

Let $\bigwedge^{d_{1}, d_{2}}$ be a column vector with $2 N-3$ components which are connection coefficients: $\bigwedge^{d_{1}, d_{2}}=\left[\Omega_{k}^{d_{1}, d_{2}}\right]_{k=1: 2 N-3}$. Equation (9) gives a system of linear equations with $\bigwedge^{d_{1}, d_{2}}$ as unknown vector; we can write (9) in vector form as

$$
\frac{1}{2^{d_{1}+d_{2}-1}} \bigwedge^{d_{1}, d_{2}}=T \bigwedge^{d_{1}, d_{2}}
$$


where $T$ is a square matrix of order $2 N-3$; that is, $T_{k: l}=$ $\sum_{m=0}^{N-1} p_{m} p_{l-2 k+m}$, where indices $k$ and $l$ vary from 1 to $2 N-3$. We use the substitution [13] $y=2^{j} x, C_{l}=2^{j / 2} c_{l}$, throughout our work. This substitution provides a way of calculating the connection coefficients. Here, $j$ and $l$ are integers and $2^{j}$ is scaling factor. It corresponds to either the expansion $(j>0)$ or the contraction $(j<0)$ of the scaling or wavelet function.

Define connection coefficients as

$$
\Omega_{k}^{0, d}=2^{d j} \int \phi^{(0)}(y) \phi^{d}(y-k) d y .
$$

Similarly, we can obtain

$$
\frac{1}{2^{d-1}} \bigwedge^{0, d}=T \bigwedge^{0, d}
$$

It is homogeneous system and thus does not have a unique nonzero solution. In order to make the system inhomogeneous, one equation is added which is derived from the moment equation of the scaling function [3] as follows:

$$
x^{d}=\sum_{i} m_{i}^{d} 2^{j / 2} \phi_{i}\left(2^{j} x\right),
$$

where $m_{i}^{d}$ is the $d$ th moment of $\phi_{j, i}(x):=\phi\left(2^{j} x-i\right)$ and we can compute it by considering the orthonormality of $\phi(x)$ that is

$$
m_{i}^{d}=\int x^{d} 2^{j / 2} \phi_{i}\left(2^{j} x\right) d x
$$

Considering the substitution $y=2^{j} x$ in (13), we arrive at

$$
y^{d}=2^{j d} 2^{j / 2} \sum_{i} m_{i}^{d} \phi_{i}(y)
$$

Differentiate (15) $d$ times to get

$$
d !=2^{j d} 2^{j / 2} \sum_{i} m_{i}^{d} \phi_{i}^{d}(y) .
$$

Taking inner product on both sides of (16) with $\phi(y-p)$,

$$
d !=2^{j / 2} \sum_{i} m_{i}^{d} 2^{j d} \int \phi_{i}^{d}(y) \phi_{p}(y) d y,
$$

or

$$
d ! 2^{-j / 2}=\sum_{i} m_{i}^{d} \bigwedge^{d, 0}
$$

Equation (14) implies

$$
\begin{gathered}
m_{i}^{d}=\int \frac{y^{d}}{2^{j d}} 2^{j / 2} \phi_{i}(y) d \frac{y}{2^{j}}, \\
m_{i}^{d}=2^{-j d-j / 2} \int y^{d} \phi_{i}(y) d y, \\
m_{i}^{d}=2^{-j d-j / 2} M_{i}^{d},
\end{gathered}
$$

where $M_{i}^{d}=\int x^{d} \phi_{i}(x) d x$ is the $d$ th moment of $\phi_{i}$ and $M_{0}^{0}=1$ implies the unit area under $\phi$. Equation (19) shows that $d$ th moment of $\phi_{j, i}(x)$ is equal to the $2^{-j d-j / 2}$ times $d$ th moment of $\phi(x-i)$.

Latto et al. [3] derive an explicit formula to compute the moments of $\phi(x)$. Consider

$$
M_{i}^{j}=\frac{1}{2\left(2^{j}-1\right)} \sum_{k=0}^{j}\left(\begin{array}{l}
j \\
k
\end{array}\right) i^{j-k} \sum_{l=0}^{k-1}\left(\begin{array}{l}
k \\
l
\end{array}\right) M_{0}^{l}\left(\sum_{i=0}^{N-1} a_{i} i^{k-1}\right),
$$

where $a_{i}$ are Daubechies wavelet coefficients. Finally, we get the system for the calculation of connection coefficients. Consider

$$
\left(\begin{array}{c}
T-\frac{1}{2^{d-1}} I \\
m^{d}
\end{array}\right) \bigwedge^{0, d}=\left(\begin{array}{c}
0 \\
d ! 2^{-j / 2}
\end{array}\right)
$$

where $m^{d}$ is a row vector with all the $m_{i}^{d}$.

\section{Implementation of Wavelet-Galerkin Method}

Consider the following form of boundary value problem:

$$
y^{\prime \prime}(x)+a y^{\prime}(x)+b y(x)=f(x), \quad y(0)=\alpha, \quad y(1)=\beta,
$$

where $a, b, \alpha$, and $\beta$ are real constants. A trial solution for (22) is

$$
y(x) \cong \sum_{l} c_{l} 2^{j / 2} \phi\left(2^{j} x-l\right), \quad l \in \mathbb{Z}
$$

Use (23) in (22) to obtain

$$
\begin{aligned}
& \frac{d^{2}}{d x^{2}} \sum_{l} c_{l} 2^{j / 2} \phi\left(2^{j} x-l\right)+a \frac{d}{d x} \sum_{l} c_{l} 2^{j / 2} \phi\left(2^{j} x-l\right) \\
& \quad+b \sum_{l} c_{l} 2^{j / 2} \phi\left(2^{j} x-l\right)-f(x) \neq 0, \quad l \in \mathbb{Z} .
\end{aligned}
$$

For simplicity, use substitution $y=2^{j} x, C_{l}=2^{j / 2} c_{l}$. Also, we have $d^{n} / d x^{n}=2^{n j} d^{n} / d y^{n}, n \in \mathbb{N}$.

Now (24) implies

$$
\begin{gathered}
\sum_{l} C_{l} 2^{2 j} \frac{d^{2}}{d y^{2}} \phi(y-l)+a \sum_{l} C_{l} 2^{j} \frac{d}{d y} \phi(y-l) \\
+b \sum_{l} C_{l} \phi(y-l)-f\left(\frac{y}{2^{j}}\right) \neq 0, \quad l \in \mathbb{Z} .
\end{gathered}
$$

Multiplying $\phi_{p}(y)$, on both sides of (25), and integrating, we get

$$
\begin{aligned}
& \sum_{l} C_{l} 2^{2 j} \int \phi(y-p) \frac{d^{2}}{d y^{2}} \phi(y-l) d y \\
& \quad+a \sum_{l} C_{l} 2^{j} \int \phi(y-p) \frac{d}{d y} \phi(y-l) d y
\end{aligned}
$$




$$
\begin{gathered}
+b \sum_{l} C_{l} \int \phi(y-p) \phi(y-l) d y \\
=\int \phi(y-p) f\left(\frac{y}{2^{j}}\right) d y,
\end{gathered}
$$

where $f(x)=\sum_{i=0}^{m} b_{i} x^{i}$, is a polynomial of degree $m$ in $x$.

The orthonormality of Daubechies wavelets implies

$$
\int \phi(y-p) \phi(y-l) d y=\delta_{p, l} .
$$

Now use (11) and (27) in (26); we have

$$
\sum_{l} C_{l}\left(\Omega_{l-p}^{0,2}+a \Omega_{l-p}^{0,1}+b \delta_{p, l}\right)=\sum_{i=0}^{m} b_{i} \int \frac{y^{i}}{2^{j i}} \phi(y-p) d y,
$$

or

$$
\sum_{l} C_{l}\left(\Omega_{l-p}^{0,2}+a \Omega_{l-p}^{0,1}+b \delta_{p, l}\right)=\sum_{i=0}^{m} \frac{b_{i}}{2^{j i}} M_{p}^{i} .
$$

Treatment of the boundary conditions [25] is as follows.

Conditions $y(0)=\alpha$ and $y(1)=\beta$ imply

$$
\begin{aligned}
& \sum_{l} C_{l} \phi_{l}(0)=\alpha, \\
& \sum_{l} C_{l} \phi_{l}(1)=\beta .
\end{aligned}
$$

Since inner product of $\phi_{l}(y)$ and $\phi_{p}(y)$ gives (27), therefore (30) implies

$$
\begin{aligned}
& \sum_{l} C_{l} \delta_{l, p}(0)=\alpha \\
& \sum_{l} C_{l} \delta_{l, p}(1)=\beta .
\end{aligned}
$$

\section{Quasilinearization}

The quasilinearization approach is a generalized NewtonRaphson technique for functional equations [26, 27]. It converges quadratically to the exact solution, if there is convergence at all, and it has monotone convergence.

Let us consider the nonlinear $n$th order differential equation as follows:

$$
y^{\prime \prime}(x)=f(y(x), x)
$$

with the boundary conditions

$$
y(a)=\alpha, \quad y(b)=\beta, \quad a \leq x \leq b .
$$

Here, $f$ is a function of the function $y(x)$. Choose an initial approximation of the function $y(x)$, let us say $y_{0}(x)$; it may be $y_{0}(x)=\alpha$, for $a \leq x \leq b$. The function $f$ can now be expanded around the function $y_{0}(x)$ by the use of the Taylor series. Consider

$$
\begin{aligned}
f(y(x), x) \approx & f\left(y_{0}(x), x\right) \\
& +\left(y(x)-y_{0}(x)\right) f_{y_{0}}\left(y_{0}(x), x\right),
\end{aligned}
$$

where second and higher order terms are ignored. Using (34) in (32), we get

$$
y^{\prime \prime}(x) \approx f\left(y_{0}(x), x\right)+\left(y(x)-y_{0}(x)\right) f_{y_{0}}\left(y_{0}(x), x\right) .
$$

Solving (35) for $y(x)$, call it $y_{1}(x)$ and expand (32) about $y_{1}(x)$

$$
y^{\prime \prime}(x) \approx f\left(y_{1}(x), x\right)+\left(y(x)-y_{1}(x)\right) f_{y_{1}}\left(y_{1}(x), x\right),
$$

we obtain a third approximation for $y(x)$, call it $y_{2}(x)$. Assume that the problem converges and continue the procedure for obtaining desired accuracy. Recurrence relation is of the form

$$
y_{r+1}^{\prime \prime}(x)=f\left(y_{r}(x), x\right)+\left(y_{r+1}(x)-y_{r}(x)\right) f_{y_{r}}\left(y_{r}(x), x\right),
$$

where $y_{r}(x)$ is known and can be used for obtaining $y_{r+1}(x)$. Equation (37) is always a linear differential equation and boundary conditions are

$$
y_{r+1}(a)=\alpha, \quad y_{r+1}(b)=\beta .
$$

Now consider the nonlinear second order differential equation of the form [26]

$$
y^{\prime \prime}(x)=f\left(y^{\prime}(x), y(x), x\right) .
$$

Here, the first derivative $y^{\prime}(x)$ can be considered as another function and (39) implies

$$
\begin{aligned}
y_{r+1}^{\prime \prime}(x)= & f\left(y_{r}^{\prime}(x), y_{r}(x), x\right) \\
& +\left(y_{r+1}(x)-y_{r}(x)\right) f_{y_{r}}\left(y_{r}^{\prime}(x), y_{r}(x), x\right) \\
& +\left(y_{r+1}^{\prime}(x)-y_{r}^{\prime}(x)\right) f_{y_{r}^{\prime}}\left(y_{r}^{\prime}(x), y_{r}(x), x\right),
\end{aligned}
$$

with the same boundary conditions

$$
y_{r+1}(a)=\alpha, \quad y_{r+1}(b)=\beta .
$$

Similarly, one can follow the same procedure for higher order nonlinear differential equations to obtain the recurrence relation

$$
\begin{aligned}
L^{n} y_{r+1}(x)= & f\left(y_{r}(x), y_{r}^{\prime}(x), \ldots, y_{r}^{n-1}(x), x\right) \\
& +\sum_{j=0}^{n-1}\left(y_{r+1}^{j}(x)-y_{r}^{j}(x)\right) \\
& \times f_{y^{j}}\left(y_{r}(x), y_{r}^{\prime}(x), \ldots, y_{r}^{n-1}(x), x\right),
\end{aligned}
$$

where $n$ is order of the differential equation. Equation (42) is always a linear differential equation and can be solved recursively, where $y_{r}(x)$ is known and one can use it to get $y_{r+1}(x)$.

In order to test the wavelet-Galerkin method with quasilinearization technique, four different nonlinear problems are considered. 


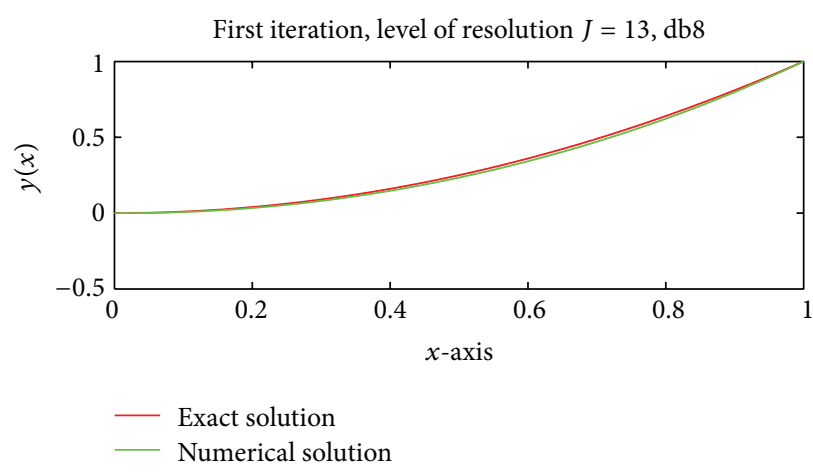

(a)

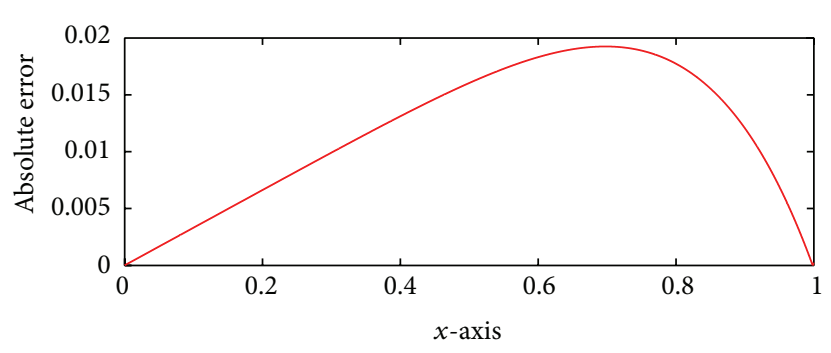

(c)

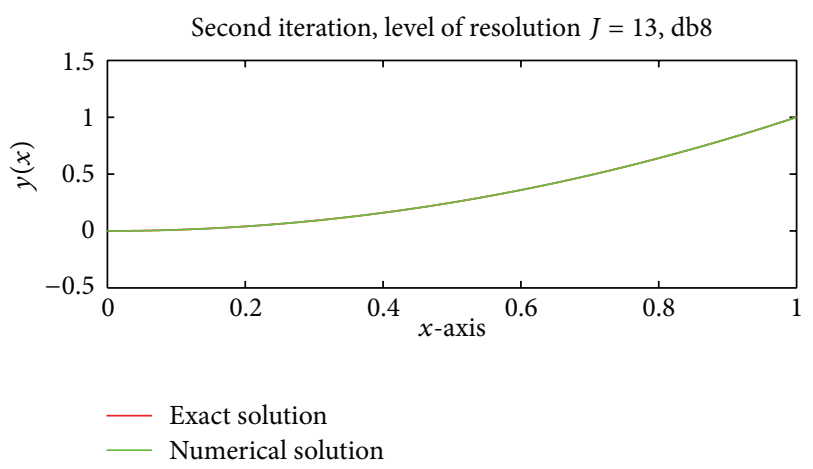

(b)

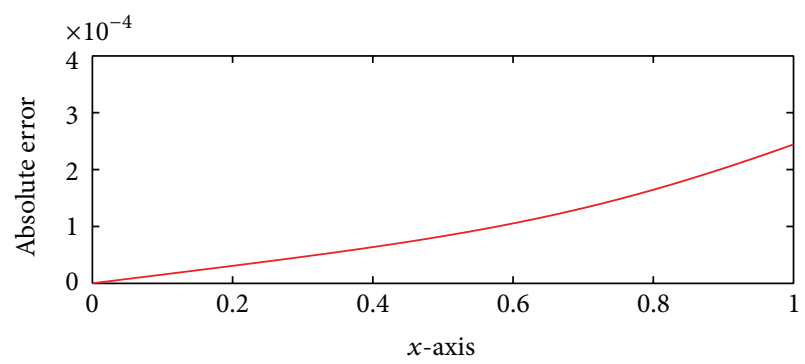

(d)

Figure 1: Comparison of exact solution and solution by wavelet-Galerkin method with quasilinearization technique at $J=13$, for different iterations, and we used D16.

TABLE 1: Comparison of exact solution $y_{\text {exact }}$ and solution by the wavelet-Galerkin method with quasilinearization technique $y_{\mathrm{NEW}}$ at second iteration: level of resolution $J=13$ and $D 16$ is used as Galerkin bases.

\begin{tabular}{lccc}
\hline $\begin{array}{l}\text { Third iteration } \\
x\end{array}$ & $J=13$ & & \\
\hline 0.1 & $y_{\mathrm{NEW}}$ & $y_{\text {exact }}$ & Absolute error \\
0.2 & $1.0022 e-2$ & $1.0 e-2$ & $2.2423 e-5$ \\
0.3 & $4.0045 e-2$ & $4.0 e-2$ & $4.4852 e-5$ \\
0.4 & $9.0067 e-2$ & $9.0 e-2$ & $6.7318 e-5$ \\
0.5 & $1.6009 e-1$ & $1.6 e-1$ & $8.9901 e-5$ \\
0.6 & $2.5011 e-1$ & $2.5 e-1$ & $1.1275 e-4$ \\
0.7 & $3.6014 e-1$ & $3.6 e-1$ & $1.3613 e-4$ \\
0.8 & $4.9016 e-1$ & $4.9 e-1$ & $1.6038 e-4$ \\
0.9 & $6.4019 e-1$ & $6.4 e-1$ & $1.8602 e-4$ \\
\hline
\end{tabular}

\section{Applications}

In this section, we solve some nonlinear ordinary differential equations by the wavelet-Galerkin method along with quasilinearization technique and compare the results with those obtained by other methods and exact solution.

Example 1. We consider the nonlinear two-point boundary value problem [28] as follows:

$$
y^{\prime \prime}(x)+y^{2}(x)=x^{4}+2, \quad 0<x<1,
$$

subject to the boundary conditions $y(0)=0, y(1)=1$. The exact solution is $y(x)=x^{2}$. Apply quasilinearization to (43); we get

$$
y_{r+1}^{\prime \prime}(x)+2 y_{r}(x) y_{r+1}(x)=y_{r}^{2}(x)+2+x^{4},
$$

with the boundary conditions $y_{r+1}(0)=0, y_{r+1}(1)=1$.

Applying wavelet-Galerkin method to (44), we have

$$
\sum_{l} C_{l}\left(\Omega_{l-p}^{0,2}+2 y_{r}(x) \delta_{p, l}\right)=y_{r}^{2}(x)+\sum_{i=0}^{4} \frac{b_{i}}{2^{j i}} M_{p}^{i},
$$

and boundary conditions imply

$$
\sum_{l} C_{l} \delta_{l, p}(0)=0, \quad \sum_{l} C_{l} \delta_{l, p}(1)=1
$$

where $b=[2,0,0,0,1]$, with the initial approximation $y_{0}(x)=0$.

We solved (43) by using D16 and fixed the level of resolution $J=13$. Figure 1 shows the exact solution and approximate solution by proposed method at first and second iteration; that is, $y_{0}(x)$ is the initial approximation, and by using $y_{0}(x)$ we get $y_{1}(x)$, that is, the solution at first iteration, and then $y_{1}(x)$ is used to get $y_{2}(x)$, which is the solution of (43) at second iteration. Table 1 is used to compare the approximate solution by proposed method at second iteration for $J=13$ and exact solution. We may get more accurate results while increasing level of resolution at higher iteration. 


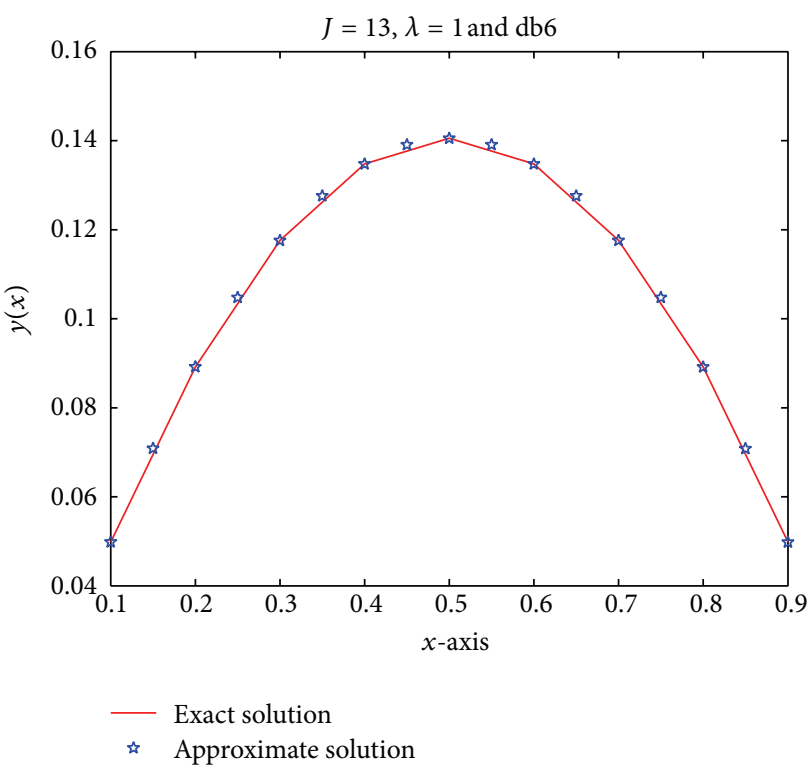

(a)

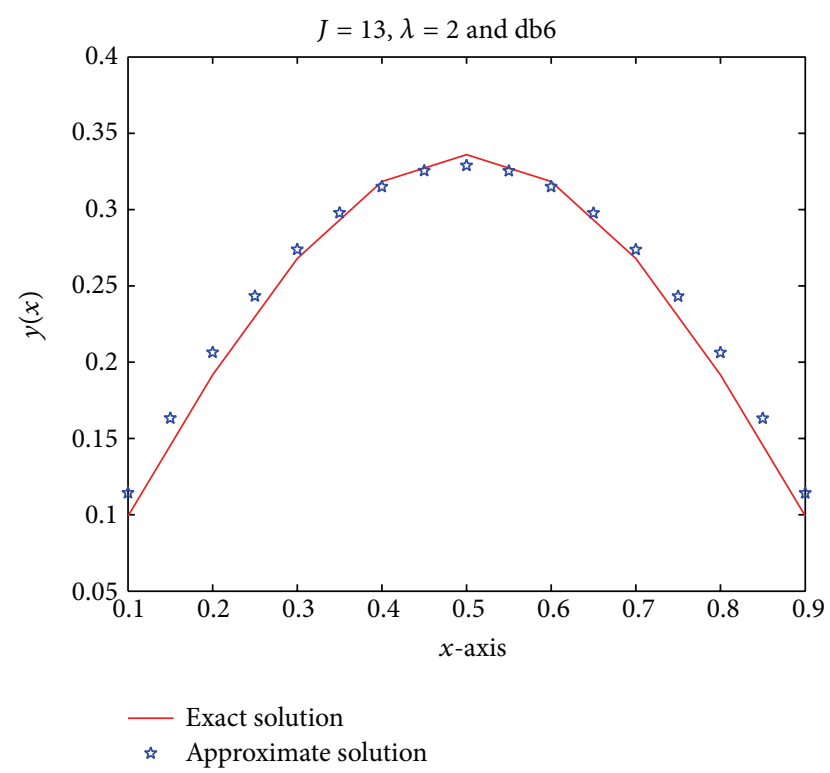

(b)

FIGURE 2: Comparison of exact solution and solution by wavelet-Galerkin method with quasilinearization technique at level of resolution $J=13$ and we use $D 12$ as Galerkin bases and at $\lambda=1$ and $\lambda=2$, respectively.

Example 2. We consider the nonlinear Bratu's boundary value problem as follows:

$$
-y^{\prime \prime}(x)=\lambda e^{y(x)}, \quad y(0)=0, \quad y(1)=0 .
$$

The quasilinear form of (47) is

$$
y_{r+1}^{\prime \prime}(x)+\lambda e^{y_{r}(x)} y_{r+1}(x)=-\lambda e^{y_{r}(x)}\left(1-y_{r}(x)\right),
$$

with the boundary conditions $y_{r+1}(0)=0, y_{r+1}(1)=0$.

Wavelet-Galerkin method for (48) implies

$$
\sum_{l} C_{l}\left(\Omega_{l-p}^{0,2}+\lambda e^{y_{r}(x)} \delta_{p, l}\right)=-\lambda e^{y_{r}(x)}\left(1-y_{r}(x)\right)
$$

and, from boundary conditions, we have

$$
\sum_{l} C_{l} \delta_{l, p}(0)=0, \quad \sum_{l} C_{l} \delta_{l, p}(1)=0
$$

with the initial approximation $y_{0}(x)=0$.

Bratu's boundary value problem is solved by using $D 12$ as Galerkin bases and at level of resolution $J=13$. Decomposition method [17] is already implemented on (47). We compared our results with the results obtained by decomposition method and exact solution. Our results are more accurate as compared to decomposition method [17] at $\lambda=1$ and $\lambda=2$ as shown in Tables 2 and 3, respectively. We used the MATLAB command of one-dimensional data interpolation using spline to get the values at $x=0.1, x=$ $0.2, \ldots, x=0.9$ and plot the exact and approximate solutions at these points for $\lambda=1$ and $\lambda=2$ as shown in Figure 2 .
TABLE 2: Comparison of exact solution $y_{\text {exact }}$, solution by decomposition method $y_{\mathrm{DM}}$, and solution by the wavelet-Galerkin method with quasilinearization technique $y_{\mathrm{NEW}}$ at second iteration and D12 is used as Galerkin bases.

\begin{tabular}{lccc}
\hline \multirow{2}{*}{$\begin{array}{c}\text { Second iteration } \\
x\end{array}$} & \multicolumn{2}{c}{$\lambda=1$} \\
\hline 0.1 & $y_{\mathrm{DM}}[17]$ & $y_{\text {NEW }}$ & $y_{\text {exact }}$ \\
0.2 & 0.0471616875 & 0.0498386633 & 0.0498467900 \\
0.3 & 0.0871680000 & 0.0891737644 & 0.0891899350 \\
0.4 & 0.1177614375 & 0.1175850602 & 0.1176090956 \\
0.5 & 0.1369920000 & 0.1347586231 & 0.1347902526 \\
0.6 & 0.1435546875 & 0.1405003497 & 0.1405392142 \\
0.7 & 0.1369920000 & 0.1347446015 & 0.1347902526 \\
0.8 & 0.1177614375 & 0.1175571769 & 0.1176090956 \\
0.9 & 0.0871680000 & 0.0891323318 & 0.0891899350 \\
& 0.0471616875 & 0.0497841328 & 0.0498467900 \\
\hline
\end{tabular}

Example 3. Consider the nonlinear Troesch's boundary value problem as follows:

$$
\begin{gathered}
y^{\prime \prime}(x)-\lambda \sinh (\lambda y(x))=0, \quad 0 \leq x \leq 1, \\
y(0)=0, \quad y(1)=1 .
\end{gathered}
$$

The quasilinearized form of (51) is

$$
\begin{aligned}
& y_{r+1}^{\prime \prime}(x)-\lambda^{2} \cosh \left(\lambda y_{r}(x)\right) y_{r+1}(x) \\
& \quad=\lambda \sinh \left(\lambda y_{r}(x)\right)-\lambda^{2} y_{r}(x) \cosh \left(\lambda y_{r}(x)\right),
\end{aligned}
$$

where $0 \leq x \leq 1$, with the boundary conditions $y_{r+1}(0)=0$, $y_{r+1}(1)=1$. 


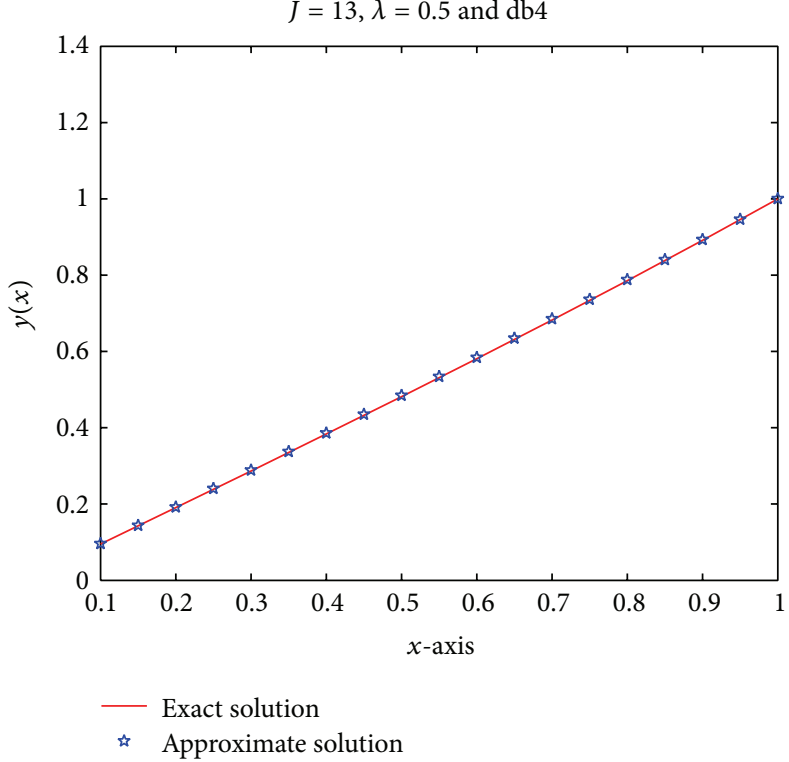

(a)

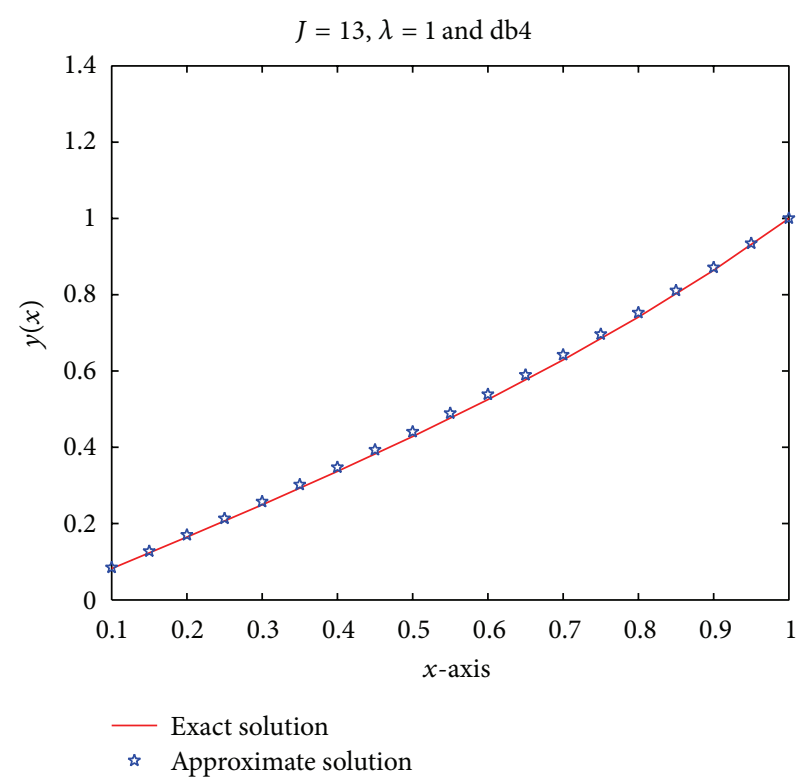

(b)

FIGURE 3: Comparison of exact solution and solution by wavelet-Galerkin method with quasilinearization technique at level of resolution $J=13$ and $D 8$ is used as Galerkin bases and at $\lambda=0.5$ and $\lambda=1$, respectively.

TABLE 3: Comparison of exact solution $y_{\text {exact }}$, solution by decomposition method $y_{\mathrm{DM}}$, and solution by the wavelet-Galerkin method with quasilinearization technique $y_{\mathrm{NEW}}$ at second iteration and $D 12$ is used as Galerkin bases.

\begin{tabular}{lccc}
\hline \multirow{2}{*}{$\begin{array}{l}\text { Second iteration } \\
x\end{array}$} & $y_{\mathrm{DM}}[17]$ & $y_{\mathrm{NEW}}$ & $y_{\text {exact }}$ \\
\hline 0.1 & 0.1144107440 & 0.1143861795 & 0.0991935000 \\
0.2 & 0.2064191156 & 0.2063705472 & 0.1917440000 \\
0.3 & 0.2738793116 & 0.2738079343 & 0.2679915000 \\
0.4 & 0.3150893646 & 0.3149970528 & 0.3183360000 \\
0.5 & 0.3289524214 & 0.3288416969 & 0.3359375000 \\
0.6 & 0.3150893646 & 0.3149632892 & 0.3183360000 \\
0.7 & 0.2738793116 & 0.2737413240 & 0.2679915000 \\
0.8 & 0.2064191156 & 0.2062728271 & 0.1917440000 \\
0.9 & 0.1144107440 & 0.1142597336 & 0.0991935000 \\
\hline
\end{tabular}

Implementation of wavelet-Galerkin method to (52) implies

$$
\begin{aligned}
& \sum_{l} C_{l}\left(\Omega_{l-p}^{0,2}-\lambda^{2} \cosh \left(\lambda y_{r}(x)\right) \delta_{p, l}\right) \\
& =\lambda \sinh \left(\lambda y_{r}(x)\right)-\lambda^{2} y_{r}(x) \cosh \left(\lambda y_{r}(x)\right),
\end{aligned}
$$

and boundary conditions lead to

$$
\sum_{l} C_{l} \delta_{l, p}(0)=0, \quad \sum_{l} C_{l} \delta_{l, p}(1)=1
$$

with the initial approximation $y_{0}(x)=0$. Tables 4 and 5 and Figure 3 represent the solution of (51) at second iteration.
TABLE 4: Comparison of exact solution $y_{\text {exact }}$, solution by variational iteration method $y_{\mathrm{VIM}}$, and solutions by the wavelet-Galerkin method with quasilinearization technique $y_{\mathrm{NEW}}$ at second iteration, at different level of resolutions, and we used $D 8$ as Galerkin bases.

\begin{tabular}{lcccc}
\hline & & \multicolumn{3}{c}{$\lambda=0.5$} \\
Second iteration & & $J=11$ & $J=13$ \\
$x$ & $y_{\text {VIM }}[18]$ & $y_{\text {NEW }}$ & $y_{\text {NEW }}$ & $y_{\text {exact }}$ \\
\hline 0.1 & 0.100042 & 0.095994 & 0.095956 & 0.095177 \\
0.2 & 0.200334 & 0.192228 & 0.192152 & 0.190634 \\
0.3 & 0.301128 & 0.288944 & 0.288829 & 0.286653 \\
0.4 & 0.402677 & 0.386385 & 0.386231 & 0.383523 \\
0.5 & 0.505241 & 0.484798 & 0.484606 & 0.481537 \\
0.6 & 0.609082 & 0.584436 & 0.584204 & 0.581002 \\
0.7 & 0.714470 & 0.685556 & 0.685284 & 0.682235 \\
0.8 & 0.821682 & 0.788424 & 0.788112 & 0.785572 \\
0.9 & 0.931008 & 0.893316 & 0.892962 & 0.891367 \\
1.0 & 1.042740 & 1.000592 & 1.000194 & 1.000000 \\
\hline
\end{tabular}

We use $D 8$ as Galerkin bases to find the solution of (51) at different level of resolutions and at $\lambda=0.5$ and $\lambda=$ 1 as shown in Tables 4 and 5, respectively. Solutions by proposed method are compared with variational iteration method [18] and with exact solution. Our results are in high agreement with exact solution and better than variational iteration method [18].

Example 4. Consider the following nonlinear boundary value problem:

$$
y^{\prime \prime}(x)+y^{\prime}(x)+y^{3}(x)+y(x)=f(x),
$$




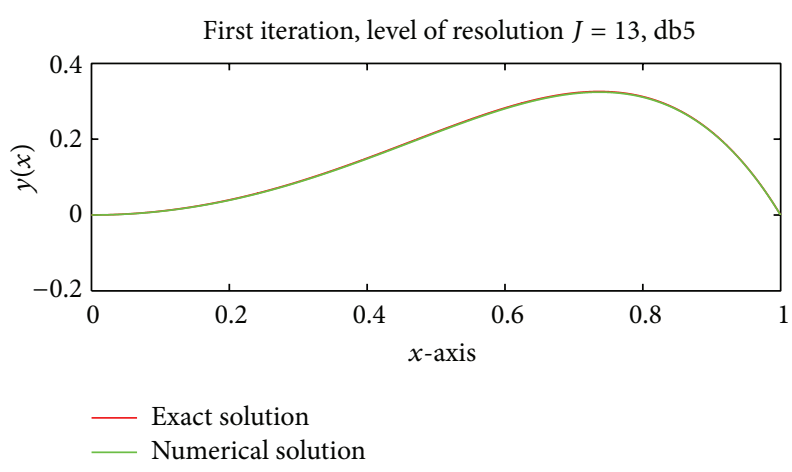

(a)

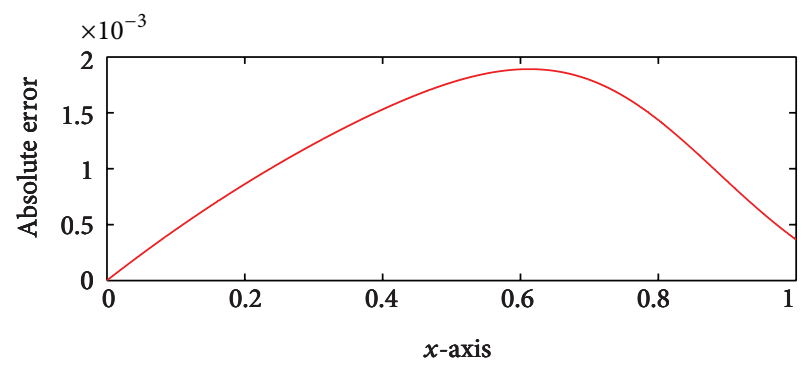

— Absolute error

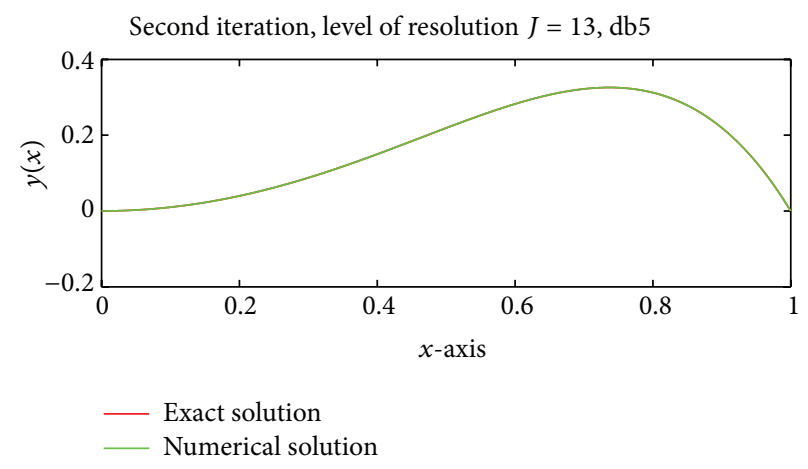

(b)

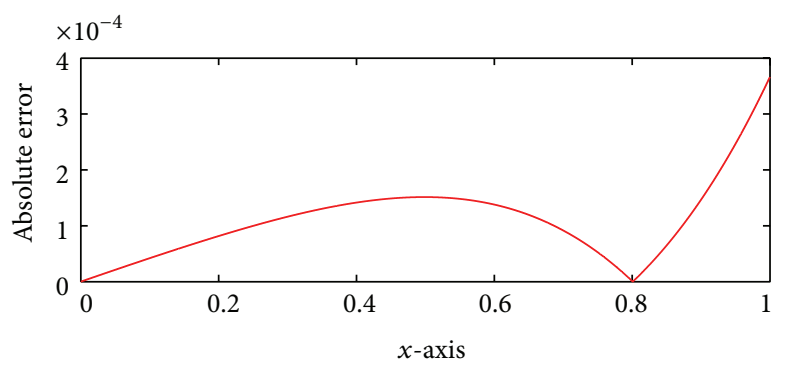

- Absolute error

(c)

(d)

FIGURE 4: Comparison of exact solution and solution by wavelet-Galerkin method with quasilinearization technique at $J=13$, for different iterations, and we use $D 10$ as Galerkin bases.

TABLE 5: Comparison of exact solution $y_{\text {exact }}$, solution by variational iteration method $y_{\mathrm{VIM}}$, and solutions by the wavelet-Galerkin method with quasilinearization technique $y_{\mathrm{NEW}}$ at second iteration, at different level of resolutions, and we used D8 as Galerkin bases.

\begin{tabular}{lcccc}
\hline \multirow{2}{*}{$\begin{array}{l}\text { Seconditeration } \\
x\end{array}$} & & $J=11$ & $J=13$ \\
& $y_{\text {VIM }}[18]$ & $y_{\text {NEW }}$ & $y_{\text {NEW }}$ & $y_{\text {exact }}$ \\
\hline 0.1 & 0.100167 & 0.084715 & 0.084674 & 0.081797 \\
0.2 & 0.201339 & 0.170280 & 0.170197 & 0.164531 \\
0.3 & 0.304541 & 0.257558 & 0.257433 & 0.249167 \\
0.4 & 0.410841 & 0.347444 & 0.347275 & 0.336732 \\
0.5 & 0.521373 & 0.440880 & 0.440666 & 0.428347 \\
0.6 & 0.637362 & 0.538877 & 0.538616 & 0.525274 \\
0.7 & 0.760162 & 0.642538 & 0.642226 & 0.628971 \\
0.8 & 0.891287 & 0.753089 & 0.752722 & 0.741168 \\
0.9 & 1.032460 & 0.871929 & 0.871495 & 0.863970 \\
1.0 & 1.185650 & 1.000718 & 1.000226 & 1.000000 \\
\hline
\end{tabular}

subject to the boundary conditions $y(0)=0, y(1)=0$, where $f(x)=2+2 x+x^{2}-20 x^{3}-5 x^{4}-x^{5}+x^{6}-3 x^{9}+3 x^{12}-x^{15}$ and exact solution is given by

$$
y(x)=x^{2}-x^{5}
$$

Applying the quasilinearization technique to (55), we get

$$
\begin{aligned}
& y_{r+1}^{\prime \prime}(x)+y_{r+1}^{\prime}(x)+\left(1+3 y_{r}^{2}(x)\right) y_{r+1}(x) \\
& \quad=f(x)+2 y_{r}^{3}(x),
\end{aligned}
$$

with the boundary conditions $y_{r+1}(0)=0, y_{r+1}(1)=0$.

Now we apply the wavelet-Galerkin method to (57) as follows:

$$
\begin{gathered}
\sum_{l} C_{l}\left(\Omega_{l-p}^{0,2}+\Omega_{l-p}^{0,1}+\left(1+3 y_{r}^{2}(x)\right) \delta_{p, l}\right) \\
=2 y_{r}^{3}(x)+\sum_{i=0}^{15} \frac{b_{i}}{2^{j i}} M_{p}^{i},
\end{gathered}
$$

and boundary conditions imply

$$
\sum_{l} C_{l} \delta_{l, p}(0)=0, \quad \sum_{l} C_{l} \delta_{l, p}(1)=0,
$$

where $b=[2,2,1,-20,-5,-1,1,0,0,-3,0,0,3,0,0,-1]$, with the initial approximations $y_{0}(x)=0$.

Numerical solution by proposed method for (55) at second iteration and level of resolution $J=13$ are stable and accurate as shown in Table 6. To get the more accurate results increase the iteration or level of resolution or both. Figure 4 shows that approximate solution converges to the exact solution while increasing iterations. Here, D10 is used as Galerkin bases. 
TABLE 6: Comparison of exact solution $y_{\text {exact }}$ and solution by the wavelet-Galerkin method with quasilinearization technique $y_{\mathrm{NEW}}$ at second iteration: level of resolutions $J=13$ and $D 10$ is used as Galerkin bases.

\begin{tabular}{lccc}
\hline $\begin{array}{l}\text { Second iteration } \\
x\end{array}$ & $J=13$ & & \\
\hline 0.1 & $y_{\text {NEW }}$ & $y_{\text {exact }}$ & Absolute error \\
0.2 & 0.0100320512 & 0.0099900000 & $4.2051152889 e-005$ \\
0.3 & 0.0397617227 & 0.0396800000 & $8.1722744304 e-005$ \\
0.4 & 0.0876864075 & 0.0875700000 & $1.1640750572 e-004$ \\
0.5 & 0.1499017545 & 0.1497600000 & $1.4175454098 e-004$ \\
0.6 & 0.2189016605 & 0.2187500000 & $1.5166050983 e-004$ \\
0.7 & 0.2823782628 & 0.2822400000 & $1.3826276219 e-004$ \\
0.8 & 0.3220219443 & 0.3219300000 & $9.1944320423 e-005$ \\
0.9 & 0.3123213602 & 0.3123199999 & $1.3602317094 e-006$ \\
\hline
\end{tabular}

\section{Conclusion}

It is shown that the proposed method, wavelet-Galerkin method with quasilinearization technique, gives stable and accurate results when applied to different nonlinear boundary value problems. The proposed method provides better and more accurate results as compared to variational iteration method and decomposition method, as shown in Tables 25. Also results are in good agreement with exact solutions. Figures 1 and 4 show that approximate solution converges to the exact solution while iterations are increased and absolute error goes down. The main advantage of the proposed method is that the different type of nonlinearities can be easily handled.

\section{Conflict of Interests}

The authors declare that there is no conflict of interests regarding the publication of this paper.

\section{Acknowledgment}

The authors are grateful to the anonymous reviewers for their valuable comments which led to the improvement of the paper.

\section{References}

[1] J. H. Mathews and K. D. Fink, Numerical Methods Using MATLAB, Prentice Hall, New York, NY, USA, 1999.

[2] G. Beylkin, "On the representation of operators in bases of compactly supported wavelets," SIAM Journal on Numerical Analysis, vol. 29, no. 6, pp. 1716-1740, 1992.

[3] A. Latto, H. L. Resnikoff, and E. Tenenbaum, "The evaluation of connection coefficients of compactly supported wavelets," in Proceedings of the French-USA Workshop on Wavelets and Turbulence, Princeton University, June 1991, Springer, New York, NY, USA.

[4] M. Chen, C. Hwang, and Y. Shih, "The computation of waveletGalerkin approximation on a bounded interval," International
Journal for Numerical Methods in Engineering, vol. 39, no. 17, pp. 2921-2944, 1996.

[5] J. M. Restrepo and G. K. Leaf, "Inner product computations using periodized Daubechies wavelets," International Journal for Numerical Methods in Engineering, vol. 40, no. 19, pp. 3557-3578, 1997.

[6] K. Amaratunga, J. R. Williams, S. Qian, and J. Weiss, "WaveletGalerkin solutions for one dimensional partial differential equations," IESL Technical Report 9205, Intelligent Engineering Systems Laboratory, M. I. T ., 1992.

[7] V. Mishra and Sabina, "Wavelet Galerkin solutions of ordinary differential equations," International Journal of Mathematical Analysis, vol. 5, no. 9, pp. 407-424, 2011.

[8] S. Jianhua, Y. Xuming, Y. Biquan, and S. Yuantong, "WaveletGalerkin solutions for differential equations," Wuhan University. Journal of Natural Sciences, vol. 3, no. 4, pp. 403-406, 1998.

[9] J. Xu and W. C. Shann, "Galerkin-Wavelet methods for twopoint boundary value problems," Numerische Mathematik, vol. 63, no. 1, pp. 123-144, 1992.

[10] S. Qian and J. Weiss, "Wavelets and the numerical solution of boundary value problems," Applied Mathematics Letters, vol. 6, no. 1, pp. 47-52, 1993.

[11] S. Qian and J. Weiss, "Wavelets and the numerical solution of partial differential equations," Journal of Computational Physics, vol. 106, no. 1, pp. 155-175, 1993.

[12] M. El-Gamel, "Comparison of the solutions obtained by Adomian decomposition and wavelet-Galerkin methods of boundary-value problems," Applied Mathematics and Computation, vol. 186, no. 1, pp. 652-664, 2007.

[13] A. K. Scheider, Implementation of Wavelet Galerkin method for boundary value problems [M.S. thesis], Rochester Institute of Technology, New York, NY, USA, 1998.

[14] R. Kalaba, "On nonlinear differential equations, the maximum operation, and monotone convergence," Journal of Mathematics and Mechanics, vol. 8, pp. 519-574, 1959.

[15] R. E. Bellman and R. E. Kalaba, Quasilinearization and Nonlinear Boundary-Value Problems, American Elsevier Publishing, New York, NY, USA, 1965.

[16] S. D. Conte and C. de Boor, Elementary Numerical Analysis, McGraw-Hill International Editions, 1981.

[17] E. Deeba, S. A. Khuri, and S. Xie, "An algorithm for solving boundary value problems," Journal of Computational Physics, vol. 159, no. 2, pp. 125-138, 2000.

[18] S. T. Mohyud-din, "Solution of Troesch's problem using He's polynomials," Revista de la Unión Matemática Argentina, vol. 52, no. 1, pp. 143-148, 2011.

[19] R. Jiwari, "A Haar wavelet quasilinearization approach for numerical simulation of Burgers' equation," Computer Physics Communications, vol. 183, no. 11, pp. 2413-2423, 2012.

[20] H. Kaur, R. C. Mittal, and V. Mishra, "Haar wavelet quasilinearization approach for solving nonlinear boundary value problems," The American Journal of Computational Mathematics, vol. 1, pp. 176-182, 2011.

[21] U. Saeed and M. Rehman, "Haar wavelet-quasilinearization technique for fractional nonlinear differential equations," Applied Mathematics and Computation, vol. 220, pp. 630-648, 2013.

[22] I. Daubechies, "Orthonormal bases of compactly supported wavelets," Communications on Pure and Applied Mathematics, vol. 41, no. 7, pp. 909-996, 1988. 
[23] I. Daubechies, Ten Lectures on Wavelets, CBMS-NSF Regional Conference Series in Applied Mathematics, SIAM, Philadelphia, Pa, USA, 1992.

[24] K. P. Soman and K. I. Ramachandran, Insight into Wavelets from Theory to Practice, PHI Learning Pvt., New Delhi, India, 2005.

[25] L. U. Dianfeng, T. Ohyoshi, and L. ZHU, Treatment of Boundary Condition in the Application of wavelet-Galerkin Method to a SH Wave Problem, Akita University, Akita, Japan, 1996.

[26] E. S. Lee, Quasilinearization and Invariant Imbedding, Academic Press, New York, NY, USA, 1968.

[27] V. B. Mandelzweig and F. Tabakin, "Quasilinearization approach to nonlinear problems in physics with application to nonlinear ODEs," Computer Physics Communications, vol. 141, no. 2, pp. 268-281, 2001.

[28] V. S. Erturk and S. Momani, "Differential transform method for obtaining positive solutions for two-point nonlinear boundary value problems," International Journal Mathematical Manuscripts, vol. 1, pp. 65-72, 2007. 


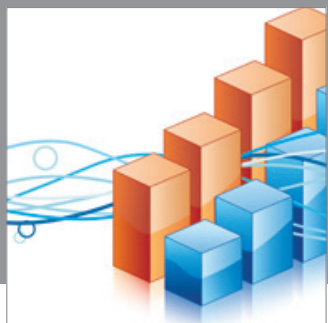

Advances in

Operations Research

mansans

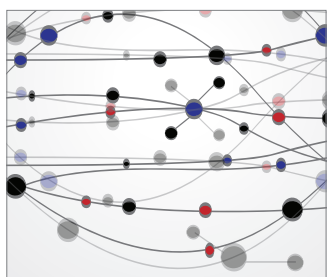

The Scientific World Journal
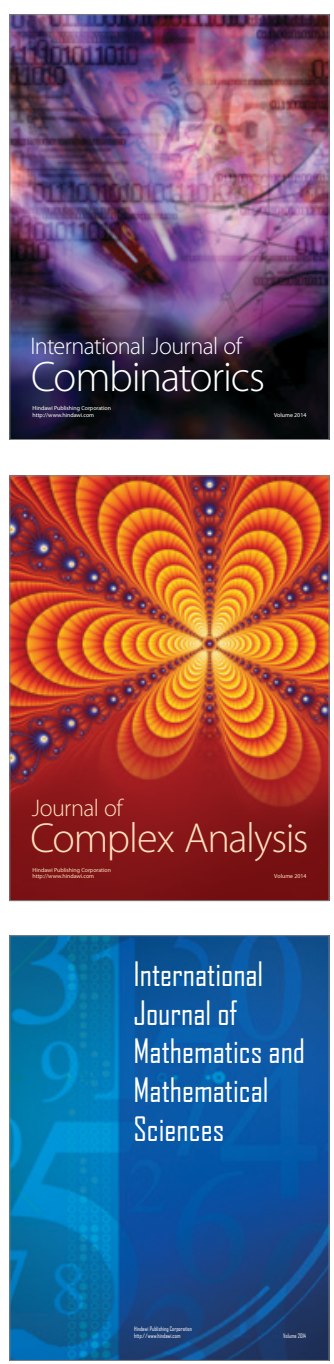
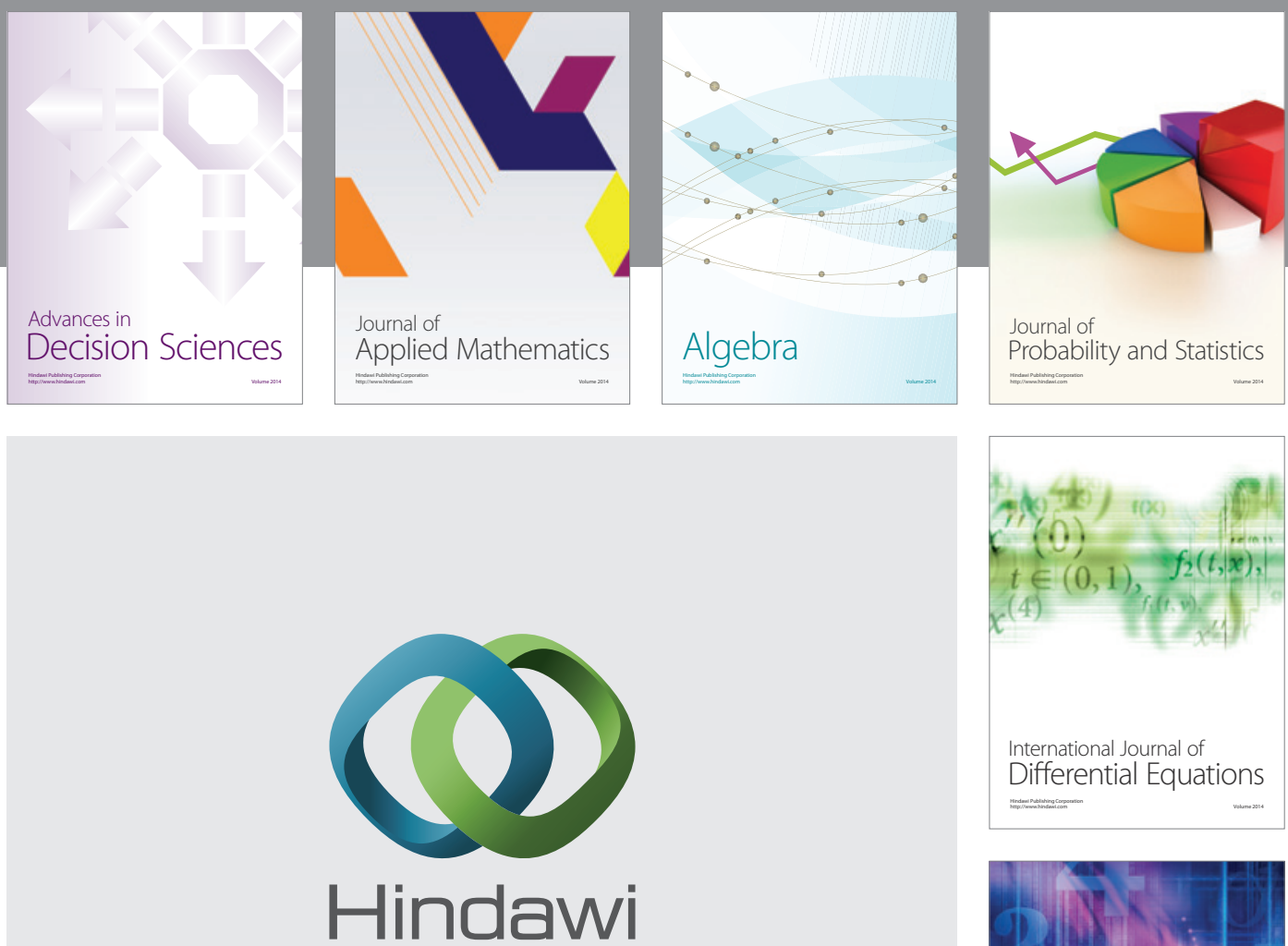

Submit your manuscripts at http://www.hindawi.com
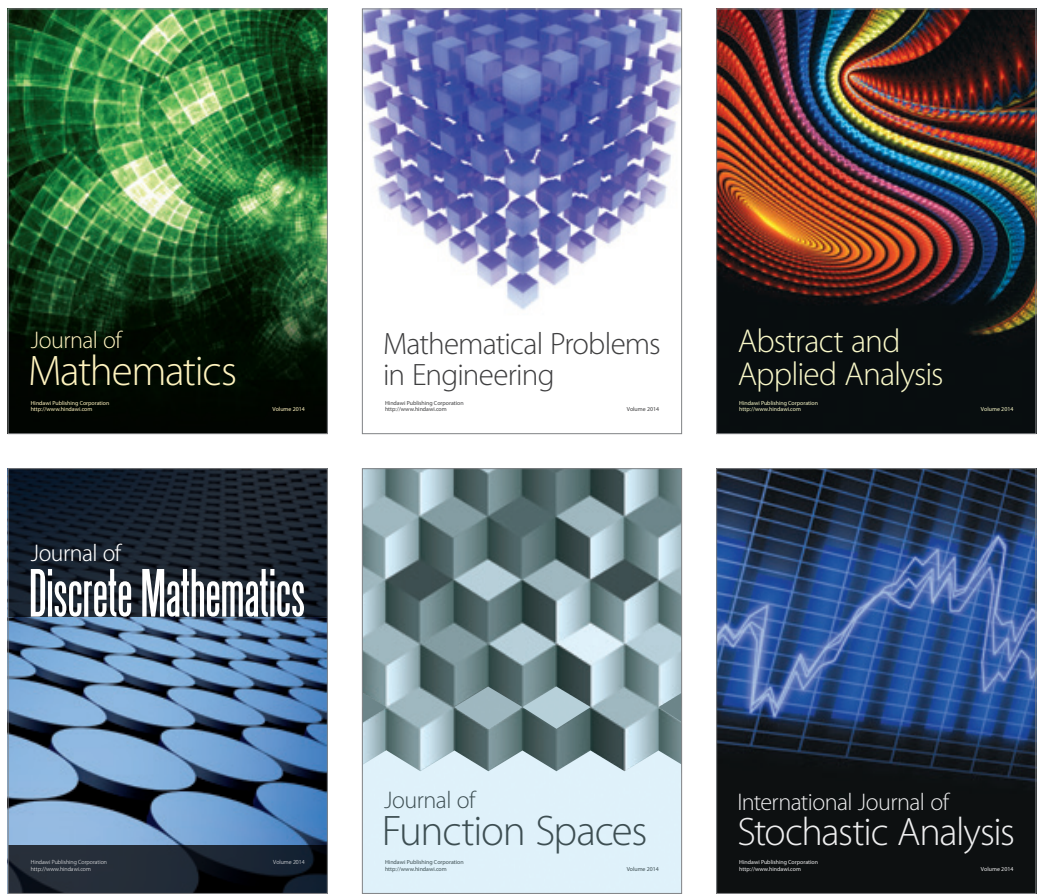

Journal of

Function Spaces

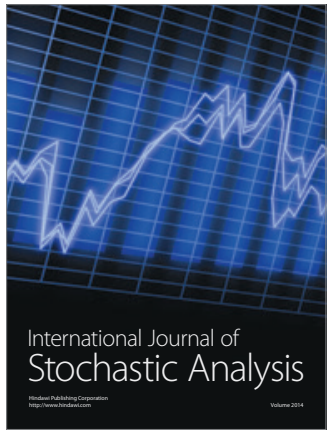

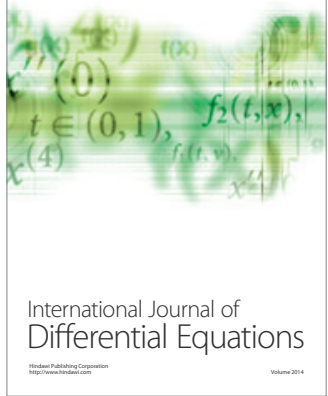
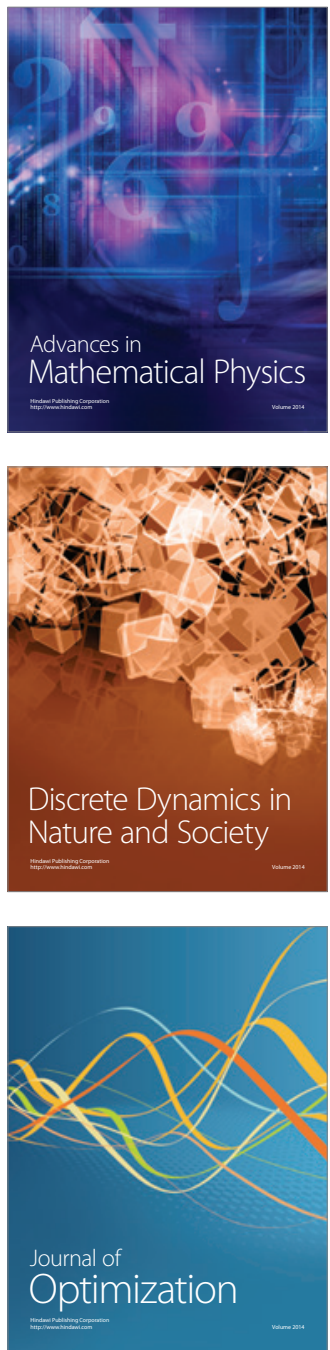